\title{
Optimal Integration for Functions of Bounded Variation*
}

\author{
By J. F. Traub and D. Lee
}

\begin{abstract}
The unique optimal information and the unique optimal linear algorithm are obtained for the integration of functions of bounded variation.
\end{abstract}

1. Introduction. For a class of real-valued functions, we seek an approximation to the integral of any function in the class, provided that the function values are given at $n$ points. A summary of what is currently known about this problem may be found in [1, Section 6.4].

In this paper, we study the class $F$ of real-valued functions of uniformly bounded variation on the unit interval. Concepts used in this paper are defined for very general settings in [1] and [2]. To aid the reader, they are defined in this paper for the special case of integration. We summarize the results of this paper.

(i) If $n$ function evaluations are used, then the intrinsic uncertainty in the integral is at least $1 / 2 n$, and $[1 / 2 \varepsilon]$ function evaluations guarantee an $\varepsilon$-approximation.

(ii) The optimal function evaluation points are $(2 i-1) / 2 n, i=1,2, \ldots, n$, and this optimal information is unique.

(iii) The optimal algorithm using the optimal information is the averaging algorithm: $(1 / n) \sum_{i=1}^{n} f((2 i-1) / 2 n)$, and this is the unique optimal linear algorithm.

(iv) The averaging algorithm is within at most one unit of being an optimal complexity algorithm.

(v) The averaging algorithm is only a constant factor better than the composite trapezoidal and Simpson algorithms.

2. Basic Concepts. A function $f$ defined on the unit interval is of bounded variation if there exists $M>0$ such that for any partition $\pi, 0 \leqslant x_{0}<x_{1}<\cdots<x_{n}<x_{n+1}$ $\leqslant 1, \sum_{i=0}^{n}\left|f\left(x_{i+1}\right)-f\left(x_{i}\right)\right|<M$. The total variation of $f$ is defined as

$$
V_{f}=\sup _{\pi} \sum_{i=0}^{n}\left|f\left(x_{i+1}\right)-f\left(x_{i}\right)\right| \text {. }
$$

We say a class $F$ of functions is of uniformly bounded variation if $F=\{f: f:[0,1] \rightarrow \mathbf{R}$ and $V_{f} \leqslant B$, where $B>0$. Without loss of generality, we take the bound $B$ to be unity.

Received August 1, 1983; revised October 15, 1984

1980 Mathematics Subject Classification. Primary 68C25, 65D30, 65D32.

* This research was supported in part by the National Science Foundation under Grant MCS-7823676 and the Advanced Research Projects Agency under Contract N00039-82-C-0427. 
We seek an approximation to $\int_{0}^{1} f(x) d x, \forall f \in F$, given function values at an $n$-partition, that is, at points $0 \leqslant x_{1}<x_{2}<\cdots<x_{n} \leqslant 1$. That is, the information $N$ is defined as $N: F \rightarrow \mathbf{R}^{n}$, and

$$
N(f)=\left[f\left(x_{1}\right), f\left(x_{2}\right), \ldots, f\left(x_{n}\right)\right] \quad \forall f \in F .
$$

We denote $x_{0}=0, x_{n+1}=1, \Delta_{i}=x_{i+1}-x_{i}$ for $i=0,1, \ldots, n$, and $\Delta=$ $\max \left\{2 \Delta_{0}, 2 \Delta_{n}, \Delta_{1}, \Delta_{2}, \ldots, \Delta_{n-1}\right\}$. We have

LEMMA 2.1. (i) $\Delta \geqslant 1 / n$; (ii) $\Delta=1 / n$ iff $\Delta_{0}=\Delta_{n}=1 / 2 n$ and $\Delta_{i}=1 / n$ for $i=1,2, \ldots, n-1$.

The proof is trivial, and is omitted.

Given information $N$ and $f \in F$, the set of indistinguishable elements from $f$ in $F$ is

$$
V(N, f)=\left\{\tilde{f} \in F: \tilde{f}\left(x_{i}\right)=f\left(x_{i}\right), i=1,2, \ldots, n\right\}
$$

The following lemma measures the uncertainty in the integral caused by indistinguishable elements.

LEMma 2.2. Let $N$ be information corresponding to an $n$-partition and let $f \in F$. Then,

$$
L \leqslant \int_{0}^{1} \tilde{f}(x) d x \leqslant U \text { for all } \tilde{f} \in V(N, f),
$$

where

$$
\begin{aligned}
U= & f\left(x_{1}\right) \Delta_{0}+f\left(x_{n}\right) \Delta_{n} \\
& +\sum_{i=1}^{n-1} \max \left\{f\left(x_{i}\right), f\left(x_{i+1}\right)\right\} \Delta_{i}+\Delta\left(1-\bar{V}_{f}\right) / 2, \text { and } \\
L= & f\left(x_{1}\right) \Delta_{0}+f\left(x_{n}\right) \Delta_{n} \\
& +\sum_{i=1}^{n-1} \min \left\{f\left(x_{i}\right), f\left(x_{i+1}\right)\right\} \Delta_{i}-\Delta\left(1-\bar{V}_{f}\right) / 2,
\end{aligned}
$$

where $\bar{V}_{f}=\sum_{i=1}^{n-1}\left|f\left(x_{i+1}\right)-f\left(x_{i}\right)\right|$. Furthermore, there exist $\tilde{f}_{L}, \tilde{f}_{U} \in V(N, f)$, such that $\int_{0}^{1} \tilde{f}_{L}(x) d x=L$ and $\int_{0}^{1} \tilde{f}_{U}(x) d x=U$.

Proof. We first show that for $\tilde{f} \in F$,

$$
\begin{aligned}
& {\left[\sup _{x_{0} \leqslant x \leqslant x_{1}} \tilde{f}(x)-\tilde{f}\left(x_{1}\right)\right]+\left[\sup _{x_{n} \leqslant x \leqslant x_{n+1}} \tilde{f}(x)-\tilde{f}\left(x_{n}\right)\right]} \\
& \quad+2 \sum_{i=1}^{n-1}\left\{\sup _{x_{i} \leqslant x \leqslant x_{i+1}} \tilde{f}(x)-\max \left\{\tilde{f}\left(x_{i}\right), \tilde{f}\left(x_{i+1}\right)\right\}\right\} \\
& \quad \leqslant 1-\bar{V}_{\tilde{f}} .
\end{aligned}
$$


For an arbitrary $\delta>0$, there exists $\xi_{i} \in\left[x_{i}, x_{i+1}\right]$ such that $\sup _{x_{i} \leqslant x \leqslant x_{i+1}} \tilde{f}(x) \leqslant$ $\tilde{f}\left(\xi_{i}\right)+\delta, i=0,1, \ldots, n$. Therefore,

$$
\begin{aligned}
& {\left[\sup _{x_{0} \leqslant x \leqslant x_{1}} \tilde{f}(x)-\tilde{f}\left(x_{1}\right)\right]+\left[\sup _{x_{n} \leqslant x \leqslant x_{n+1}} \tilde{f}(x)-\tilde{f}\left(x_{n}\right)\right]} \\
& +2 \sum_{i=1}^{n-1}\left\{\sup _{x_{i} \leqslant x \leqslant x_{i+1}} \tilde{f}(x)-\max \left\{\tilde{f}\left(x_{i}\right), \tilde{f}\left(x_{i+1}\right)\right\}\right\}+\sum_{i=1}^{n-1}\left|\tilde{f}\left(x_{i+1}\right)-\tilde{f}\left(x_{i}\right)\right| \\
& \leqslant\left[\tilde{f}\left(\xi_{0}\right)-\tilde{f}\left(x_{1}\right)\right]+\left[\tilde{f}\left(\xi_{n}\right)-\tilde{f}\left(x_{n}\right)\right] \\
& +\sum_{i=1}^{n-1}\left\{2\left[\tilde{f}\left(\xi_{i}\right)-\max \left\{\tilde{f}\left(x_{i}\right), \tilde{f}\left(x_{i+1}\right)\right\}\right]+\left|\tilde{f}\left(x_{i+1}\right)-\tilde{f}\left(x_{i}\right)\right|\right\}+2 n \delta \\
& \leqslant\left|\tilde{f}\left(\xi_{0}\right)-\tilde{f}\left(x_{1}\right)\right|+\left|\tilde{f}\left(\xi_{n}\right)-\tilde{f}\left(x_{n}\right)\right| \\
& +\sum_{i=1}^{n-1}\left[\left|\tilde{f}\left(\xi_{i}\right)-\tilde{f}\left(x_{i}\right)\right|+\left|\tilde{f}\left(x_{i+1}\right)-\tilde{f}\left(\xi_{i}\right)\right|\right]+2 n \delta \\
& \leqslant V_{\tilde{f}}+2 n \delta \leqslant 1+2 n \delta .
\end{aligned}
$$

Since $\delta$ is arbitrary,

and (2.5) follows.

$$
\begin{aligned}
& {\left[\sup _{x_{0} \leqslant x \leqslant x_{1}} \tilde{f}(x)-\tilde{f}\left(x_{1}\right)\right]+\left[\sup _{x_{n} \leqslant x \leqslant x_{n+1}} \tilde{f}(x)-\tilde{f}\left(x_{n}\right)\right] } \\
& \quad+2 \sum_{i=1}^{n-1}\left\{\sup _{x_{i} \leqslant x \leqslant x_{i+1}} \tilde{f}(x)-\max \left\{\tilde{f}\left(x_{i}\right), \tilde{f}\left(x_{i+1}\right)\right\}\right\} \\
&+\sum_{i=1}^{n-1}\left|\tilde{f}\left(x_{i+1}\right)-\tilde{f}\left(x_{i}\right)\right| \leqslant 1
\end{aligned}
$$

Let $\tilde{f} \in V(N, f)$, then

$$
\begin{aligned}
& \int_{0}^{1} \tilde{f}(x) d x \leqslant \sum_{i=1}^{n}\left[\sup _{x_{i} \leqslant x \leqslant x_{i+1}} \tilde{f}\left(x_{i}\right)\right] \Delta_{i} \\
&=\tilde{f}\left(x_{1}\right) \Delta_{0}+\tilde{f}\left(x_{n}\right) \Delta_{n}+\sum_{i=1}^{n-1} \max \left\{\tilde{f}\left(x_{i}\right), \tilde{f}\left(x_{i+1}\right)\right\} \Delta_{i} \\
&+\left[\sup _{x_{0} \leqslant x \leqslant x_{1}} \tilde{f}(x)-\tilde{f}\left(x_{1}\right)\right] \Delta_{0}+\left[\sup _{x_{n} \leqslant x \leqslant x_{n+1}} \tilde{f}(x)-\tilde{f}\left(x_{n}\right)\right] \Delta_{n} \\
&+\sum_{i=1}^{n-1}\left\{\sup _{x_{i} \leqslant x \leqslant x_{i+1}} \tilde{f}(x)-\max \left\{\tilde{f}\left(x_{i}\right), \tilde{f}\left(x_{i+1}\right)\right\}\right\} \Delta_{i} \\
& \leqslant \tilde{f}\left(x_{1}\right) \Delta_{0}+\tilde{f}\left(x_{n}\right) \Delta_{n}+\sum_{i=1}^{n-1} \max \left\{\tilde{f}\left(x_{i}\right), \tilde{f}\left(x_{i+1}\right)\right\} \Delta_{i} \\
&+\frac{\Delta}{2}\left\{\sup _{x_{0} \leqslant x \leqslant x_{1}} \tilde{f}(x)-\tilde{f}\left(x_{1}\right)\right]+\left[\begin{array}{c}
\left.\sup _{x_{n} \leqslant x \leqslant x_{n+1}} \tilde{f}(x)-\tilde{f}\left(x_{n}\right)\right] \\
\end{array}\right. \\
&\left.+2 \sum_{i=1}^{n-1}\left\{\sup _{x_{i} \leqslant x \leqslant x_{i+1}} \tilde{f}(x)-\max \left\{\tilde{f}\left(x_{i}\right), \tilde{f}\left(x_{i+1}\right)\right\}\right\}\right\} \\
& \leqslant \tilde{f}\left(x_{1}\right) \Delta_{0}+\tilde{f}\left(x_{n}\right) \Delta_{n}+\sum_{i=1}^{n-1} \max \left\{\tilde{f}\left(x_{i}\right), \tilde{f}\left(x_{i+1}\right)\right\} \Delta_{i}+\Delta\left(1-\bar{V}_{\tilde{f}}\right) / 2 .
\end{aligned}
$$


The last step follows from (2.5). Therefore,

$$
\begin{aligned}
\int_{0}^{1} \tilde{f}(x) d x & \leqslant \tilde{f}\left(x_{1}\right) \Delta_{0}+\tilde{f}\left(x_{n}\right) \Delta_{n}+\sum_{i=1}^{n-1} \max \left\{\tilde{f}\left(x_{i}\right), \tilde{f}\left(x_{i+1}\right)\right\} \Delta_{i}+\Delta\left(1-\bar{V}_{\tilde{f}}\right) / 2 \\
& =f\left(x_{1}\right) \Delta_{0}+f\left(x_{n}\right) \Delta_{n}+\sum_{i=1}^{n-1} \max \left\{f\left(x_{i}\right), f\left(x_{i+1}\right)\right\} \Delta_{i}+\Delta\left(1-\bar{V}_{f}\right) / 2,
\end{aligned}
$$

i.e., $\int_{0}^{1} \tilde{f}(x) d x \leqslant U$. The result for $L$ follows from that for $U$ by replacing $f$ by $-f$.

Let

$$
I= \begin{cases}{\left[0, x_{1}\right)} & \text { if } 2 \Delta_{0}=\Delta, \\ \left(x_{i}, x_{i+1}\right) & \text { if } 2 \Delta_{0}<\Delta \text { and } i=\min \left\{j: \Delta_{j}=\Delta \text { and } 1 \leqslant j \leqslant n-1\right\}, \\ \left(x_{n}, 1\right] & \text { if } 2 \Delta_{0}<\Delta, \Delta_{j}<\Delta \text { for } 1 \leqslant j \leqslant n-1 \text { and } 2 \Delta_{n}=\Delta .\end{cases}
$$

Let

$$
f_{\max }(x)= \begin{cases}f\left(x_{1}\right) & \text { if } 0 \leqslant x \leqslant x_{1} \\ f\left(x_{n}\right) & \text { if } x_{n} \leqslant x \leqslant 1 \\ f\left(x_{i}\right) & \text { if } x=x_{i}, i=2,3, \ldots, n-1, \\ \max \left\{f\left(x_{i}\right), f\left(x_{i+1}\right)\right\} & \text { if } x_{i}<x<x_{i+1}, i=1,2, \ldots, n-1 .\end{cases}
$$

Finally, let

$$
\tilde{f}_{U}(x)= \begin{cases}f_{\max }(x)+\left(1-\bar{V}_{f}\right) / C_{I} & \text { if } x \in I \\ f_{\max }(x) & \text { otherwise }\end{cases}
$$

where

$$
C_{I}= \begin{cases}2 & \text { if } I=\left(x_{i}, x_{i+1}\right) \text { for some } i, 1 \leqslant i \leqslant n-1 \\ 1 & \text { if } I=\left[0, x_{1}\right) \text { or } I=\left(x_{n}, 1\right] .\end{cases}
$$

It can be verified that $V_{\tilde{f}_{U}}=1, \tilde{f}_{U} \in V(N, f)$, and $\int_{0}^{1} \tilde{f}_{U}(x) d x=U$. An analogous conclusion holds for $\tilde{f}_{L}$.

3. Optimal Information. From Lemma 2.2 we know that for all $\tilde{f} \in V(N, f)$, the integral of $\tilde{f}, \int_{0}^{1} \tilde{f}(x) d x$, is confined to the interval $[L, U]$. We call

$$
r(N, f)=(U-L) / 2 \text {, }
$$

the local radius of information $N$ at $f$. From (2.4) we have

$$
r(N, f)=\frac{1}{2}\left\{\sum_{i=1}^{n-1}\left|f\left(x_{i+1}\right)-f\left(x_{i}\right)\right| \Delta_{i}+\Delta\left(1-\bar{V}_{f}\right)\right\} .
$$

We define

$$
r(N)=\sup _{f \in F} r(N, f)
$$

as the global radius of information. The quantity $r(N, f)$ measures the intrinsic uncertainty of the integral of $f$, caused by indistinguishable elements in $V(N, f)$, and $r(N)$ measures that of the worst $f$ in $F$. We estimate the local and global radii of information in

LEMMA 3.1. Let $N$ be information corresponding to the $n$-partition $0=x_{0} \leqslant x_{1} \leqslant x_{2}$ $<\cdots<x_{n} \leqslant x_{n+1}=1$. Then,

$$
r(N, f) \leqslant \Delta / 2 \text { for all } f \in F,
$$


and

$$
r(N)=\Delta / 2 \text {. }
$$

Proof. Since $\Delta_{i} \leqslant \Delta$ for $i=1,2, \ldots, n-1$, by (3.2),

$$
\begin{aligned}
r(N, f) & =\frac{1}{2}\left[\sum_{i=1}^{n-1}\left|f\left(x_{i+1}\right)-f\left(x_{i}\right)\right| \Delta_{i}+\Delta\left(1-\bar{V}_{f}\right)\right] \\
& \leqslant \frac{\Delta}{2}\left[\sum_{i=1}^{n-1}\left|f\left(x_{i+1}\right)-f\left(x_{i}\right)\right|+\left(1-\bar{V}_{f}\right)\right]=\Delta / 2,
\end{aligned}
$$

i.e., $r(N, f) \leqslant \Delta / 2$, proving (3.4). Let $f \equiv 0$. Then by (3.2), $r(N, 0)=\Delta / 2$, i.e., $r(N)=\sup _{f \in F} r(N, f)=r(N, 0)=\Delta / 2$, proving (3.5).

The information $N(f)$ in (2.1) is said to be of cardinality $n$. Let $\Psi(n)$ be the class of all information of cardinality $n$, and let $r(n)=\inf _{N \in \Psi(n)} r(N)$. Then information $N \in \Psi(n)$ is called nth optimal if $r(N)=r(n)$. An $n$th optimal information $N$ has the minimum radius of information, among all information in $\Psi(n)$.

Let $N^{*}$ be information corresponding to the partition points $x_{i}=(2 i-1) / 2 n$, where $i=1,2, \ldots, n$, and $n \geqslant 2$. We have

THEOREM 3.1. $N^{*}$ is the unique nth optimal information with $r\left(N^{*}\right)=r(n)=$ $1 / 2 n$.

Proof. For the information $N^{*}, \Delta=1 / n$. By (3.5), $r\left(N^{*}\right)=1 / 2 n$. On the other hand, for an arbitrary $N \in \Psi(n), r(N)=\Delta / 2 \geqslant 1 / 2 n=r\left(N^{*}\right)$, by Lemma 2.1(i), and the equality holds iff $N=N^{*}$, by Lemma 2.1(ii).

Remark 3.1. (i) If the class of integrands $F_{1}$ consists of functions with a uniformly bounded first derivative, then (see [1, Section 6.4]) $N^{*}$ is an $n$th optimal information with $r\left(N^{*}\right)=1 / 4 n$.

(ii) To define information in (2.1), the partition points $x_{i}$ are independent of function values at the previously chosen partition points. This is nonadaptive information. If partition points are chosen sequentially, depending on the function values at the previously chosen partition points, we have adaptive information. For integration of functions of bounded variation, the integrand belongs to a balanced convex set. Therefore, one gains nothing by using adaptive information. For the proof, see [1, Section 2.7] or [2, Section 4.3].

4. Optimal Algorithm. Usually, one cannot compute the integral of a function exactly, and instead seeks an approximation to the integral using an algorithm $\varphi$

$$
\varphi: N(F) \rightarrow \mathbf{R} \text {. }
$$

We define the local algorithm error of $f$ as

$$
e(\varphi, N, f)=\sup _{\tilde{f} \in V(N, f)}\left|\int_{0}^{1} \tilde{f}(x) d x-\varphi(N(f))\right|,
$$

and the global algorithm error as

$$
e(\varphi, N)=\sup _{f \in F} e(\varphi, N, f) .
$$

For a given $f \in F$, the integrals of indistinguishable elements $\tilde{f} \in V(N, f)$ are in the interval [ $L, U$ ], where the sharp bounds $L$ and $U$ are given in (2.4). Therefore, for an arbitrary algorithm $\varphi, e(\varphi, N, f) \geqslant(U-L) / 2$, which by (3.1) is the local 
radius of information $r(N, f)$. Thus, we have

$$
e(\varphi, N, f) \geqslant r(N, f),
$$

and

$$
e(\varphi, N) \geqslant r(N) \text { for all } \varphi \text {. }
$$

Therefore, $r(N, f)$ and $r(N)$ are the lower bounds of local and global algorithm errors, respectively.

We present an algorithm, called the central algorithm, by choosing the center of $[L, U]$ as $\varphi^{c}(N(f))$ :

$$
\varphi^{c}(N(f))=(U+L) / 2 .
$$

Then $e\left(\varphi^{c}, N, f\right)=(U-L) / 2=r(N, f)$, and $e\left(\varphi^{c}, N\right)=r(N)$. Since $\varphi^{c}$ has the minimal $e\left(\varphi^{c}, N\right)$ among all algorithms, it is called an optimal error algorithm.

From (2.4), we have

$$
\begin{aligned}
& e\left(\varphi^{c}, N, f\right)=\frac{1}{2}\left[\sum_{i=1}^{n-1}\left|f\left(x_{i+1}\right)-f\left(x_{i}\right)\right| \Delta_{i}+\Delta\left(1-\bar{V}_{f}\right)\right], \\
& e\left(\varphi^{c}, N\right)=\Delta / 2 \\
& \varphi^{c}(N(f))=f\left(x_{1}\right) \Delta_{0}+f\left(x_{n}\right) \Delta_{n}+\sum_{i=1}^{n-1} \frac{f\left(x_{i}\right)+f\left(x_{i+1}\right)}{2} \Delta_{i},
\end{aligned}
$$

or

$$
\begin{aligned}
\varphi^{c}(N(f))= & f\left(x_{1}\right)\left(\Delta_{0}+\Delta_{1} / 2\right)+\sum_{i=2}^{n-1} f\left(x_{i}\right) \frac{\Delta_{i-1}+\Delta_{i}}{2} \\
& +f\left(x_{n}\right)\left(\Delta_{n}+\frac{\Delta_{n-1}}{2}\right) .
\end{aligned}
$$

An algorithm is linear if it is of the form

$$
\varphi(N(f))=\sum_{i=1}^{n} f\left(x_{i}\right) H_{i} .
$$

Therefore, the central algorithm is linear. We summarize the above in

THEOREM 4.1. Given information $N$, the central algorithm $\varphi^{c}$ is a linear optimal error algorithm, with local and global algorithm error equal to the local and global radius of information, respectively.

Remark 4.1. It is true in general (see [2, Sections 1.3 and 1.4]) that the central algorithm is optimal and that the local and global algorithm error of the central algorithm are equal to the local and global radius of information, respectively.

Given the unique $n$th optimal information $N^{*}$, we compare the algorithm error of the central algorithm with those of other linear algorithms in

THEOREM 4.2. The central algorithm $\varphi^{c}$ using the unique nth optimal information $N^{*}$ is

$$
\varphi^{c}\left(N^{*}(f)\right)=\frac{1}{n} \sum_{i=1}^{n} f\left(\frac{2 i-1}{2 n}\right) .
$$

Furthermore, the linear optimal error algorithm is unique. 
Proof. Let $\varphi$ be an arbitrary noncentral linear algorithm, with $\varphi\left(N^{*}(f)\right)=$ $\sum_{i=1}^{n} f\left(x_{i}\right) H_{i}$, and let $p$ be the largest subscript of $H$ such that $H_{p} \neq 1 / n$. Let

$$
f_{p}(x)= \begin{cases}0 & \text { if } x<x_{p} \\ 1 & \text { if } x \geqslant x_{p}\end{cases}
$$

Then we have

$$
\begin{aligned}
e\left(\varphi, N^{*}, f_{p}\right) & =\sup _{\tilde{f} \in V\left(N, f_{p}\right)}\left|\sum_{i=1}^{n} f_{i} H_{i}-\int_{0}^{1} f(x) d x\right| \\
& =\max \left\{\left|H_{p}+\frac{n-p}{n}-U\right|,\left|H_{p}+\frac{n-p}{n}-L\right|\right\} \\
& =\max \left\{\left|H_{p}-\frac{1}{n}-\frac{1}{2 n}\right|,\left|H_{p}-\frac{1}{n}+\frac{1}{2 n}\right|\right\} \\
& \geqslant\left|H_{p}-\frac{1}{n}\right|+\frac{1}{2 n}>\frac{1}{2 n}=e\left(\varphi^{c}, N^{*}\right) .
\end{aligned}
$$

Therefore, $e\left(\varphi, N^{*}\right) \geqslant e\left(\varphi, N^{*}, f_{p}\right)>e\left(\varphi^{c}, N^{*}\right)$ and $\varphi$ is not optimal.

5. Complexity. Given information $N$, we seek an algorithm $\varphi$ to compute an $\varepsilon$-approximation to the integral of any functions in $F$, with algorithm error $e(\varphi, N)$ $\leqslant \varepsilon$, where $\varepsilon>0$. We use the $n$th optimal information $N^{*}$ and the central algorithm $\varphi^{c}$ to obtain an $\varepsilon$-approximation. Then, from Theorem 3.1 and Theorem 4.1, we have $e\left(\varphi^{c}, N^{*}\right)=r\left(N^{*}\right)=r(n)=1 / 2 n \leqslant \varepsilon$. Therefore, $n=\lceil 1 / 2 \varepsilon\rceil$. It is obvious that $\lceil 1 / 2 \varepsilon]$ is the minimal number of function evaluations for which we can have an $\varepsilon$-approximation to the integral of any functions in $F$.

Assume that the cost of each arithmetic operation is 1 and that of each function evaluation is $c$. We first compute $N(f)=y=\left(y_{1}, \ldots, y_{n}\right)$ with information complexity $c n$, where $n$ is the cardinality of $N$. We then compute $\varphi(y)$ with combinatory complexity $\operatorname{comp}(\varphi(y))$. The complexity of algorithm $\varphi$ is thus $\operatorname{comp}(\varphi, N)=c n+$ $\sup _{f \in F} \operatorname{comp}(\varphi(y))$. By (4.12) we have

$$
\operatorname{comp}\left(\varphi^{c}, N^{*}\right)=(c+1)\left\lceil\frac{1}{2 \varepsilon}\right\rceil .
$$

We define the problem complexity of an $\varepsilon$-approximation as

$$
\operatorname{comp}(\varepsilon)=\inf _{\varphi, N}\{\operatorname{comp}(\varphi, N): e(\varphi, N) \leqslant \varepsilon\}
$$

and an optimal complexity algorithm $\varphi^{*}$ as

$$
\operatorname{comp}\left(\varphi^{*}, N\right)=\operatorname{comp}(\varepsilon) \text {, and } e\left(\varphi^{*}, N\right) \leqslant \varepsilon \text { for some } N \text {. }
$$

As noted at the beginning of this section, $n=\lceil 1 / 2 \varepsilon]$ is the minimal number of function evaluations to guarantee an $\varepsilon$-approximation. Thus, the information complexity is no less than $c[1 / 2 \varepsilon]$, and the combinatory complexity is no less than $\lceil 1 / 2 \varepsilon\rceil-1$. Therefore $\operatorname{comp}(\varepsilon) \geqslant(c+1)\lceil 1 / 2 \varepsilon\rceil-1$. Comparing this with (5.1), we notice that the difference between $\operatorname{comp}\left(\varphi^{c}, N^{*}\right)$ and $\operatorname{comp}(\varepsilon)$ is at most 1 . We propose the following

CONJECTURE. The central algorithm using the optimal information is the optimal complexity algorithm, that is,

$$
\operatorname{comp}\left(\varphi^{c}, N^{*}\right)=\operatorname{comp}(\varepsilon) \text {. }
$$


6. Comparison With Other Algorithms. We estimate the global algorithm error and algorithm complexity of some linear algorithms; the proofs are routine and are omitted.

(i) Another Riemann Sum. Let the partition points be $x_{i}=(i-1) / n, i=$ $1,2, \ldots, n$, and $\Delta=2 / n$.

$$
\begin{gathered}
\varphi(N(f))=\frac{1}{n} \sum_{i=1}^{n} f\left(\frac{i-1}{n}\right) . \\
e(\varphi, N)=\frac{1}{n} .
\end{gathered}
$$

The algorithm complexity for an $\varepsilon$-approximation is

$$
\operatorname{comp}(\varphi, N)=(c+1)\left\lceil\frac{1}{\varepsilon}\right\rceil \text {. }
$$

(ii) Composite Trapezoidal Rule. Let the partition points be $x_{i}=(i-1) /(n-1)$, $i=1,2, \ldots, n$ and $\Delta=1 /(n-1)$.

$$
\begin{gathered}
\varphi(N(f))=\frac{1}{2(n-1)}[f(0)+f(1)]+\frac{1}{n-1} \sum_{i=2}^{n-1} f\left(\frac{i-1}{n-1}\right) . \\
e(N, \varphi)=\frac{1}{n-1} .
\end{gathered}
$$

The algorithm complexity for an $\varepsilon$-approximation is

$$
\operatorname{comp}(\varphi, N)=(c+1)\left\lceil\frac{1}{\varepsilon}\right\rceil+c+2 \text {. }
$$

(iii) Composite Simpson's Rule. Assume that $n=2 m+1$. Let the partition points be $x_{i}=(i-1) / 2 m, i=1,2, \ldots, 2 m+1$, and $\Delta=1 / 2 m$.

$$
\begin{gathered}
\varphi(N(f))=\frac{1}{6 m}[f(0)+f(1)]+\frac{1}{3 m} \sum_{i=2}^{m} f\left(\frac{i-1}{m}\right)+\frac{2}{3 m} \sum_{i=1}^{m} f\left(\frac{2 i-1}{2 m}\right), \\
e(\varphi, N)=\frac{2}{3(n-1)} .
\end{gathered}
$$

The algorithm complexity for an $\varepsilon$-approximation is

$$
\operatorname{comp}(\varphi, N)=(c+1)\left\lceil\frac{2}{3 \varepsilon}\right\rceil+c+3 .
$$

Observe that the costs of the linear algorithms (i)-(iii) are within a constant factor of $\operatorname{comp}(\varepsilon)$.

Acknowledgment. We are grateful to Dr. Ivan Huerta for his valuable comments.

Department of Computer Science and Mathematics

Columbia University

New York, New York 10027

Department of Computer Science

Columbia University

New York, New York 10027

1. J. F. Traub \& H. Woźniakowski, A General Theory of Optimal Algorithms, Academic Press, New York, 1980.

2. J. F. Traub, G. W. Wasilkowski \& H. Woźniakowski, Information, Uncertainty, Complexity, Addison-Wesley, Reading, Mass., 1983. 